Review Article

\title{
Effectiveness, Safety, and Barriers to Early Mobilization in the Intensive Care Unit
}

\author{
Gopala Krishna Alaparthi $\mathbb{D},{ }^{1}$ Aishwarya Gatty $\mathbb{D D}^{2}{ }^{2}$ Stephen Rajan Samuel $\mathbb{D},{ }^{2}$ \\ and Sampath Kumar Amaravadi $\mathbb{D}^{2,3}$ \\ ${ }^{1}$ Department of Physiotherapy, College of Health Sciences, University of Sharjah, Sharjah, UAE \\ ${ }^{2}$ Department of Physiotherapy, Kastruba Medical College Mangalore, Manipal Academy of Higher Education, Mangaluru, \\ Karnataka, India \\ ${ }^{3}$ Department of Physiotherapy, College of Health Sciences, Gulf Medical University, Ajman, UAE
}

Correspondence should be addressed to Aishwarya Gatty; ashgatty95@gmail.com

Received 28 July 2020; Accepted 12 November 2020; Published 26 November 2020

Academic Editor: Timothy Plackett

Copyright ( 2020 Gopala Krishna Alaparthi et al. This is an open access article distributed under the Creative Commons Attribution License, which permits unrestricted use, distribution, and reproduction in any medium, provided the original work is properly cited.

Purpose. Patients admitted to the intensive care unit (ICU) are generally confined to bed leading to limited mobility that may have detrimental effects on different body systems. Early mobilization prevents or reduces these effects and improves outcomes in patients following critical illness. The purpose of this review is to summarize different aspects of early mobilization in intensive care. Methods. Electronic databases of PubMed, Google Scholar, ScienceDirect, and Scopus were searched using a combination of keywords. Full-text articles meeting the inclusion criteria were selected. Results. Fifty-six studies on various aspects such as the effectiveness of early mobilization in various intensive care units, newer techniques in early mobilization, outcome measures for physical function in the intensive care unit, safety, and practice and barriers to early mobilization were included. Conclusion: Early mobilization is found to have positive effects on various outcomes in patients with or without mechanical ventilation. The newer techniques can be used to facilitate early mobilization. Scoring systems-specific to the ICU—are available and should be used to quantify patients' status at different intervals of time. Early mobilization is not commonly practiced in many countries. Various barriers to early mobilization have been identified, and different strategies can be used to overcome them.

\section{Introduction}

Patients with critical illness are patients with a life-threatening disease or trauma. Such patients are at an increased risk of developing serious complications from their condition [1]. They are admitted to the intensive care unit (ICU) and are usually confined to bed which could negatively affect their mobility [2]. Prolonged immobilization, mechanical ventilation, and sedation in the course of critical illness have been associated with restricted joint mobility, muscle weakness, pressure sores, critical illness neuropathies or ICU-acquired weakness, deep vein thrombosis (DVT), long duration of mechanical ventilation, cognitive impairments, and psychological disturbances $[2,3]$.

The damaging effects of bed rest are not only limited to the musculoskeletal system but also affects other body systems. Changes noticed within the cardiac system include tachycardia, postural hypotension, decreased peak uptake of oxygen, stroke volume, and cardiac output caused due to fluid loss [4]. A decrease in ventilatory volume and secretion clearance is caused due to a supine position, resulting in atelectasis and pneumonia $[2,4]$. Residual problems which include reduced physical function and decreased quality of life are frequently experienced by critical illness survivors [5].

Earlier, bed rest was considered as a treatment for critical illness, but it also had its harmful effects [6, 7]. In 1899, Ries found that bed rest could lead to ill effects in the postoperative period, whereas the length of hospital stay could be shortened from days or weeks to hours using early mobilization [8]. In the following years, similar results were seen in patients who underwent other surgeries and in females in 
the postnatal period [9]. Also, conferences and peerreviewed journals addressed the topic of ill effects due to rest in bed [10]. Eventually, the advantages of early mobilization in mechanically ventilated patients were specified in studies $[11,12]$. Early mobilization has been tried out as a form of rehabilitation since the early nineteenth century and with a wide array of reported findings of the approach that give it a robust foundation in therapeutic rehabilitation [10].

Early mobilization is the early application and intensification of physical rehabilitation given to patients with critical illness, commenced within the initial two to five days of critical illness [13]. It includes activities such as in-bed mobility activities, range of motion exercises, sitting, standing, transfers, and gait training [4]. However, there is no agreed definition for early mobilization in mechanically ventilated patients, and what activities constitute it are poorly understood [14]. Early mobility in the ICU has been proposed to limit or prevent physical and cognitive dysfunction and provide various benefits $[13,15]$. Increase in regional ventilation, perfusion, diffusion, tidal volume, minute ventilation, the efficiency of respiratory mechanics, pulmonary immune factors, mucociliary transport, and airway clearance and decrease in airflow resistance are the changes seen in the pulmonary system [16].

Cardiovascular system changes include an increase in venous return, stroke volume, heart rate, myocardial contractility, cardiac output, coronary perfusion, circulating blood volume, peripheral blood flow, chest tube drainage, and peripheral tissue oxygen extraction. Neurological effects include an increase in level of consciousness and stimulus to breathe. Increased urinary output due to an increase in glomerular filtration is seen [16]. Involving patients in early mobilization may have positive effects such as reduction in muscle atrophy, delirium, and duration of mechanical ventilation, shorter length of ICU stay, enhanced physical function, and quality of life [17].

Considering the benefits of early mobilization, this study aims to review the available evidence on various aspects of early mobilization in the intensive care unit. Understanding its various aspects can aid its implementation in clinical practice and may help in achieving improved patient outcomes.

\section{Materials and Methods}

2.1. Study Selection Criteria. Full-text articles on early mobilization of patients - with or without mechanical ventilation-in the intensive care unit, published in the English language from January 2012 to April 2020 were included. Studies conducted in the paediatric intensive care unit, studies on early mobilization outside ICU, study protocols, and poster presentations were excluded.

2.2. Literature Search. Electronic databases of PubMed, Google Scholar, ScienceDirect, and Scopus were searched using combination of keywords "Early Mobilization," "Early Rehabilitation," "Intensive Care Unit," "Critically Ill patients" "Effectiveness," "Surgical," "Neurological,"
"Cardiac," "Barriers," "Outcomes," "Physical Function," "Mobility," "Safety," "Adverse effects," "Practice," "Cycling," "Electrical Muscle Stimulation," "Combilizer," and "Hydrotherapy." Synonyms were checked to exhaust the possibility of more keywords. The Boolean operator "AND” was used. References lists of the selected articles were manually reviewed. The data extraction is summarized in Figure 1.

\section{Results and Discussion}

Fifty-six studies were included in the review. Out of these, twenty studies were on the effectiveness of early mobilization in various intensive care units, ten studies on newer techniques for early mobilization, nine studies on outcomes for measuring physical function in the intensive care unit, four studies on safety, eight studies about practice, and five studies on barriers to early mobilization.

3.1. Effectiveness of Early Mobilization in the Intensive Care Unit. Over the last few years, studies have analyzed the effectiveness of early mobilization on short-term and longterm outcomes in patients with critical illness [17-26].

3.1.1. Short-Term Impact on Outcomes. Zhang et al. [18] found that early mobilization of critically ill patients reduced the incidence of ICU-acquired weakness, improved functional capacity, decreased days on mechanical ventilation, and increased number of patients who could stand and rate of discharge from the ICU.

The systematic review by Doiron et al. [19] reviewed the studies on early mobilization during or after mechanical ventilation versus delayed mobilization or usual care in critically ill patients. It was inconclusive due to the low quality of the included studies.

A meta-analysis carried out by Zang et al. [20] found early mobilization in critically ill to be effective in reducing ICU-acquired weakness and length of ICU stay and in preventing deep vein thrombosis, ventilator-associated pneumonia, and pressure sores. It also improved functional mobility. Similarly, Zhang et al. [17] reviewed the effects of early mobilization for critically ill mechanically ventilated patients and reported that it could improve outcomes such as shortening the duration of mechanical ventilation and decreasing the length of stay in the ICU.

A review by Tipping et al. [21] found that active mobilization in the ICU caused improvement in body function and participation which was measured using muscle strength and walking ability. A systematic review and metaanalysis by Castro-Avila et al. [22] reported that early rehabilitation in patients admitted to the intensive care unit/ high dependency unit for more than forty-eight hours improved patients' walking ability at hospital discharge but did not have any effect on muscle strength and functional status.

Most of these reviews had low quality evidence due to reasons such as small sample size, heterogeneity in the population, intervention, and outcome measures. Therefore, good quality studies were suggested to verify the results 


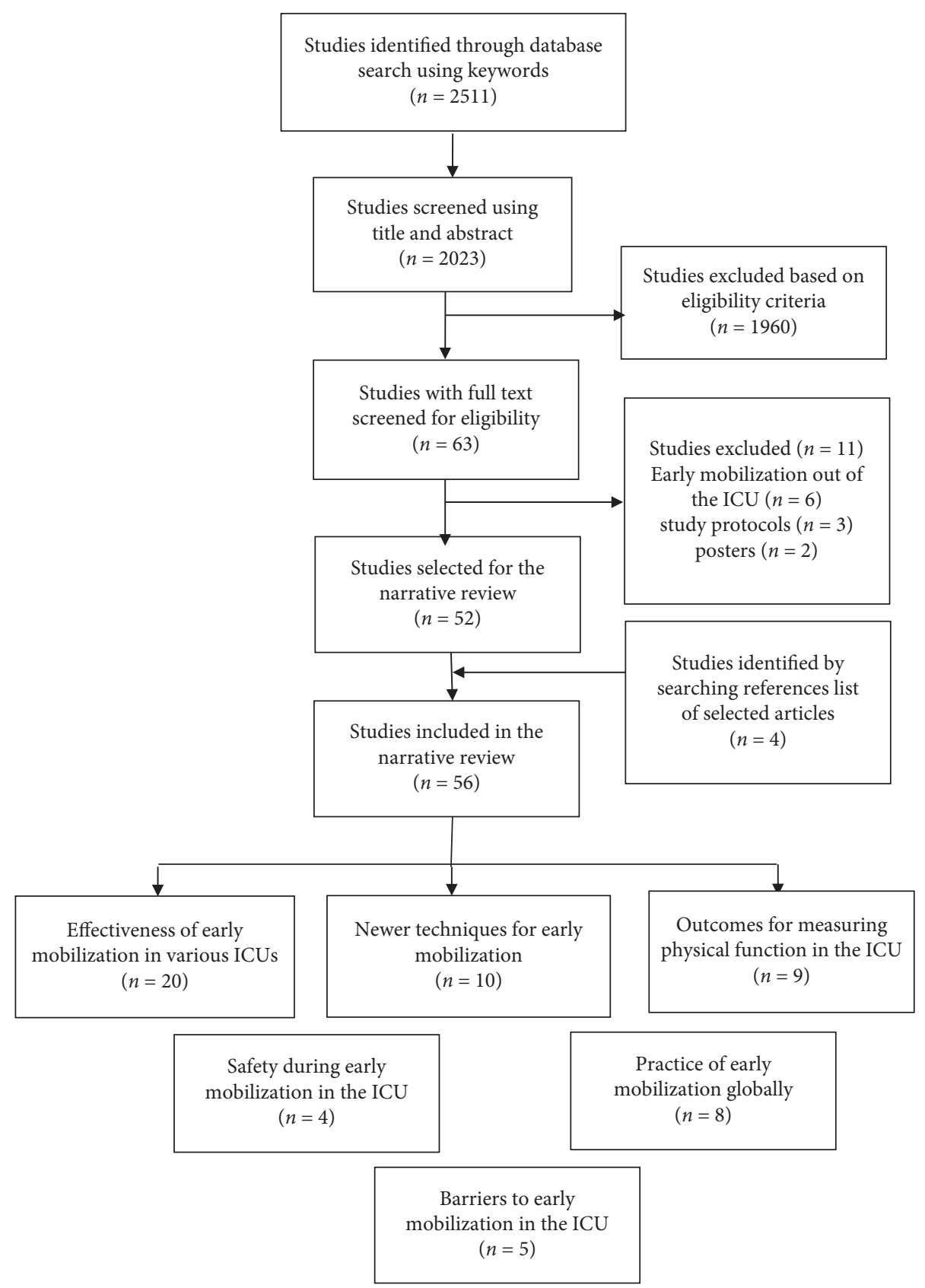

FIgURE 1: Flow diagram showing data extraction.

[17-22]. The need for further studies was advised to determine the effects of different early mobilization protocols and to find out the most effective and safe one [17]. Shortterm impacts on outcomes are summarized in Table 1.

3.1.2. Long-Term Impact on Outcomes. Okada et al. [23] conducted a systematic review and meta-analysis to study the effects of delayed versus early mobilization in critically ill adult patients and found no differences between the two groups regarding mortality and health-related quality of life. As the review included studies with limited sample size and heterogeneous definition of interventions, further studies were suggested to confirm these findings. According to Castro-Avila et al. [22], early mobilization did have an impact on the quality of life. Zhang et al. [18] did not find any effect on mortality. Tipping et al. [21] found that early mobilization did not decrease the mortality of patients.

Effects of intensive, twice daily, upright mobilization was studied on mechanically ventilated patients by Amundadottir et al. [24]. They found no difference in outcomes between the twice-daily mobilization and daily mobilization groups at three, six, and twelve months. A randomized controlled trial by Denehy et al. [25] reported no significant difference in outcomes between intervention and usual care groups after a 12-month follow-up. Another randomized 
TABLE 1: Short-term impact of early mobilization on outcomes.

\begin{tabular}{|c|c|c|c|c|c|c|}
\hline \multirow[b]{2}{*}{ Outcomes } & \multicolumn{6}{|c|}{ Studies } \\
\hline & $\begin{array}{c}\text { Zhang et al. } \\
{[18]}\end{array}$ & $\begin{array}{c}\text { Doiron et al. } \\
{[19]}\end{array}$ & $\begin{array}{c}\text { Zang et al. } \\
{[20]}\end{array}$ & $\begin{array}{c}\text { Zhang et al. } \\
\text { [17] }\end{array}$ & $\begin{array}{c}\text { Tipping et al. } \\
{[21]}\end{array}$ & $\begin{array}{c}\text { Castro-avila } \\
\text { et al. [22] }\end{array}$ \\
\hline Muscle strength & No increase & Inconclusive & $\begin{array}{c}\text { Prevented } \\
\text { weakness }\end{array}$ & - & Improved & No difference \\
\hline $\begin{array}{l}\text { Complications such as deep vein } \\
\text { thrombosis, pneumonia, and pressure } \\
\text { sores }\end{array}$ & - & - & $\begin{array}{l}\text { Incidence } \\
\text { reduced }\end{array}$ & - & - & - \\
\hline Delirium & - & Inconclusive & - & - & - & - \\
\hline Length of ICU stay & - & Inconclusive & Decreased & Decreased & $\begin{array}{l}\text { Could not be } \\
\text { analyzed }\end{array}$ & No difference \\
\hline Length of hospital stay & - & Inconclusive & Decreased & $\begin{array}{c}\text { No } \\
\text { difference }\end{array}$ & - & - \\
\hline Duration of mechanical ventilation & Decreased & Inconclusive & No effect & Shortened & $\begin{array}{l}\text { Could not be } \\
\text { analyzed }\end{array}$ & - \\
\hline Functional capacity & Improved & & Improved & - & - & No effect \\
\hline Physical function & - & Inconclusive & - & - & - & - \\
\hline Walking ability & - & & - & - & Improved & Improved \\
\hline Discharge to home rate & Increased & & - & - & - & - \\
\hline Death in ICU & - & Inconclusive & No difference & - & - & - \\
\hline Mortality at hospital discharge & - & - & - & - & No difference & - \\
\hline
\end{tabular}

TABLE 2: Long-term impact of early mobilization on outcomes.

Studies

Outcomes

\begin{tabular}{lcc} 
& Quality of life & Mortality \\
\hline Okada et al. [23] & No difference between delayed and early mobilization & No difference between delayed and early mobilization \\
Zhang et al. [18] & groups & No decrease in 28 days mortality rate \\
Doiron et al. [19] & - & Inconclusive \\
Tipping et al. [21] & No difference at 6 months & No difference \\
Castro-Avila et al. [22] & No effect & - \\
Amundadottir et al. [24] & $\begin{array}{l}\text { No difference between intensive, twice-daily } \\
\text { mobilization, and daily mobilization groups }\end{array}$ & $\begin{array}{c}\text { No difference between intensive, twice-daily } \\
\text { mobilization, and daily mobilization groups }\end{array}$ \\
$\begin{array}{l}\text { Denehy et al. [25] } \\
\text { Wright et al. [26] }\end{array}$ & $\begin{array}{l}\text { No difference at } 12 \text { months } \\
\text { No difference at } 6 \text { months }\end{array}$ & - \\
\hline
\end{tabular}

controlled trial conducted by Wright et al. [26] concluded that the intervention group, which received a higher dosage of mobilization, did not seem to show improvement in physical outcomes at 6 months compared to standard physical rehabilitation who received usual mobilization. Loss of follow up was one of the important limitations which could have affected the results in both these studies $[25,26]$. Table 2 shows the long-term impact of early mobilization on quality of life and mortality.

\subsubsection{Effectiveness of Early Mobilization in Subpopulations of Patients with Critical Illness}

(1) Early Mobilization in the Surgical Intensive Care Unit. In patients undergoing surgeries, especially abdominal and thoracic surgeries' postoperative complications causing significant morbidity and mortality, increased medical consumption, and increased hospital stay are common. Some of these complications include atelectasis, pneumonia, acute respiratory distress syndrome, and deep vein thrombosis [27]. The reasons for these complications are altered respiratory mechanics, reduced lung volumes, respiratory muscle dysfunction, retention of secretions, changes in oxygenation, immobility, and recumbent position postsurgery. Early mobilization can aid in preventing or minimizing these complications [16].

Castelino et al. [27] conducted a systematic review on the effectiveness of early mobilization on postoperative outcomes following thoracic and abdominal surgery. The quality of included studies was found to be poor, and the results were conflicting. The study was inconclusive.

Schaller et al. [28] conducted a randomized controlled trial to find the effectiveness of early goal-directed mobilization in the surgical intensive care unit and found it to be useful as it improved the mobility of the patients at discharge and decreased the length of ICU stay. As the study included only the surgical patients who were mechanically ventilated for more than forty-eight hours, the results of this study could not be generalized to nonsurgical or nonventilated patients.

Zomorodi et al. [29] developed an early mobilization protocol for patients in surgical and trauma ICU. It was found that the protocol was successful and decreased the 
length of ICU stay. As this was a pilot study, authors suggested that further studies with a larger sample size should be performed to establish the feasibility and efficacy of this protocol.

(2) Early Mobilization in the Cardiac Intensive Care Unit. Cardiac surgeries include surgical procedures for pathologies of the heart and have significant effects causing a change in the physiological mechanisms of patients in different ways. This may lead to critical postoperative conditions that require intensive care to establish a functional recovery [30]. Cardiac surgeries present some typical complications such as acute myocardial infarction and low cardiac output syndrome $[30,31]$. Some of the other complications are mechanical ventilation for more than forty-eight hours after surgery, acute respiratory distress syndrome, pleural effusion, hypoxemia, acute respiratory failure, phrenic nerve palsy, ventilation-associated pneumonia, cerebrovascular accident, infection at surgical sites, hemorrhage, and changes in the serum electrolytes level [30].

One of the well-established contributing factors to postoperative complications is bed rest or immobility. Despite this, bed rest after surgery was being prescribed for cardiac surgery patients to reduce cardiac overload. Nevertheless, recent evidence shows numerous benefits of early mobilization postsurgery [32]. Studies have shown early mobilization to reduce postoperative outcomes [31,32].

A systematic review by Santos et al. [32] reported that early mobilization in patients after cardiac surgery prevented postoperative complications, decreased length of hospital stay, and improved functional capacity when compared with no treatment. The most effective protocol could not be found when different techniques and duration of mobilization were compared.

Moradian et al. [31] conducted a randomized controlled trial to study the effect of early mobilization on pulmonary complications after coronary artery bypass graft (CABG) and found a lower incidence of atelectasis, pleural effusion, and improved oxygenation in the intervention group. They suggested that further studies should be performed for identifying appropriate initiating time, frequency, intensity, and duration of early mobilization.

(3) Early Mobilization in the Neurological Intensive Care Unit. Acute cerebrovascular accident, subarachnoid, parenchymal and subdural hemorrhage, central nervous system infection, status epilepticus, brain tumors, neuromuscular disorders, and cerebral vascular malformation are common conditions managed in the neurological intensive care unit [33].

They are put on prolonged bed rest for the adequacy of blood flow to the brain resulting in deconditioning and electrolyte imbalance, which augments the already damaging neurological injury [33]. Sympathetic functions are altered, contributing to orthostatic hypotension after long-term bed rest [34]. Patients with severe brain injuries, such as head trauma, large brain infarcts, and subarachnoid hemorrhage may have severe cardiovascular manifestations such as arrhythmias, myocardial ischemia, hypertension, and pulmonary edema. They are also at risk of secondary brain injury because of edema and delayed vasospasm $[33,34]$.

Early mobilization is considered to be an essential aspect of care, which leads to improved outcomes [35]. Cognitive impairment, hemiparesis or hemiplegia, fluctuating intracerebral pressure and cerebral perfusion, and dislodgement of cerebral monitoring or other indwelling devices can compromise safety during mobilization [33]. Various studies on different neurological conditions have been performed to check the effectiveness of early mobilization [33-38].

Klein et al. [33] conducted a comparative study to assess the effects of early mobilization in improving mobility and clinical outcomes in the neurological ICU and found an increase in the patients' highest level of mobility without causing any severe complications.

Rocca et al. [34] studied the changes in the sympathetic system due to early mobilization with three methods: standard mobilization, gradual postural variations with robot Erigo consisting of a tilting table integrated with leg movement system, and cycling with MOTOmed consisting of an automatic leg mobilization system in supine. They found that both the new methods caused sympathetic stimulation and can be used for early mobilization, but leg movements with MOTOmed caused an increased level of catecholamine, indicating stress, and hence should be used with caution. As the sample size was small and heterogeneous, further studies are required to confirm the results of this study.

Alamri et al. [36] conducted a study to check the effectiveness of an early mobility protocol for patients diagnosed with stroke in the ICU. The patients were divided into three categories, which included unstable and on the ventilator, cooperative and on the ventilator, and cooperative and being weaned off from the ventilator. They were treated with different protocols. Early mobility protocols had positive effects on muscle strength and quality of life. No adverse events occurred; so, they were considered safe to be practiced.

Diserens et al. [37] studied the effectiveness of early mobilization in comparison with delayed mobilization in subjects with moderate to severe acute ischemic stroke and reported an apparent decrease in severe medical complications with early mobilization. Cerebral blood flow on transcranial Doppler and neurological scales showed that the protocol was safe. However, this study was performed on a small sample size with unequal dropouts, and Doppler could be performed only in $60 \%$ of the planned instances.

A study was conducted by Bartolo et al. [38] to determine the influence of early mobilization on functional outcomes in patients with severe acquired brain injury and was found to have a positive influence on clinical and functional recovery of the patients.

3.2. Effectiveness of Newer Techniques. Various techniques such as electrical muscle stimulation and cycling are being used in early mobilization. Studies have been performed to check the effectiveness of these techniques [39-48]. 
3.2.1. Electrical Muscle Stimulation. Electrical muscle stimulation (EMS) can be used as a substitute for the reversal of muscle weakness and deconditioning as applying EMS around the muscle fibers and at the neuromuscular junction generates contractions that prevent atrophy of muscles, improve circulation of blood, and alleviate the effects of long periods of immobility without overloading the cardiovascular system. These benefits may persevere for up to four to six weeks after the completion of the treatment $[39,40]$.

Falavigna et al. [39] conducted a randomized clinical trial to assess the effects of early EMS on the ankle joint range of movement and circumference of the thigh and legs in critically ill mechanically ventilated patients. It showed that EMS was effective in preserving amplitude of the ankle joint movement, increasing mobility and function, but the strength and cross-sectional area of the muscle stimulated did not increase. This could have been due to low intensity and duration of stimulation. Neuromuscular stimulation (NMES) was found to be effective in preserving the thickness of the chest and abdominal muscles in critically ill patients in a study by Acqua et al. [41].

Fischer et al. [42] studied the effect of neuromuscular stimulation in patients after cardiothoracic surgery and concluded that it did not affect the muscle layer thickness and functional outcomes but contributed to higher regain in muscle strength during the ICU stay. Also, a review by Baron et al. [40] suggested that neuromuscular stimulation in the intensive care unit has positive effects and is safe to be used.

3.2.2. Cycling. Effect of cycle ergometry in early mobilization postcardiac surgery was studied in a randomized controlled trial by Gama Lordello et al. [43]. They concluded that it was safe to use but did not cause any significant difference in independent physical activity in the intervention group when compared to a standard care group.

Machado et al. [44] assessed the effects of passive cycling along with conventional physical therapy on muscle strength of peripheral muscle, number of days on a mechanical ventilator, and length of hospital stay in patients admitted to the ICU. Early mobilization using passive cycling improved peripheral muscle strength in mechanically ventilated patients with no significant changes in the number of days on the mechanical ventilator or length of hospital stay.

The effects of early mobilization using a bedside cycle ergometer in addition to conventional physical therapy were evaluated by Santos et al. [45] in a randomized controlled trial. Thickness and architecture of the quadriceps were evaluated in critically ill patients receiving invasive mechanical ventilation. No significant difference was found in these outcomes.

3.2.3. Cycling and Electrical Muscle Stimulation. Fossat et al. [46] found that early in-bed cycling exercise and electrical muscle stimulation for quadriceps did not cause any significant change in global muscle strength at discharge from the ICU when compared to usual care. In addition, there were no significant differences in secondary outcomes such as the number of ventilator-free days, ICU mobility score, or quality of life at 6 months.

3.2.4. Sara Combilizer. The Sara Combilizer is a combination of a tilt table and chair, which can be made completely horizontal to allow transfer through a sliding board and also allows standing positions to be attained. McWilliams et al. [47] assessed the effectiveness of the Sara Combilizer in facilitating safe and early mobilization of critically ill patients and found a reduction in time required for mobilization. It may be a beneficial adjunct to early mobilization protocols.

3.2.5. Hydrotherapy. Felten-Barentsz et al. [48] conducted a study to determine the feasibility and safety of hydrotherapy in critically ill patients who were mechanically ventilated. An individualized tailored program, which could include standing, walking, backstroke swimming, and moving upper limbs, was used. Any adverse events or contamination of pool water were noted. They found hydrotherapy to be feasible and safe and also concluded that further studies need to be performed to assess its cost-effectiveness and benefits.

3.3. Outcome Measures for Assessment of the Effectiveness of Early Mobilization. An outcome measure is any characteristic or quality measured to assess a patient's status. They are used to objectively determine the baseline function of a patient at the beginning of treatment and to determine the progress and treatment efficacy [49]. Various studies have used different outcomes for measuring the effectiveness of early mobilization and are mentioned in Table 3 [17-26].

Physical function impairment is a noteworthy problem faced by critical illness survivors. Measuring impairments provide information about the patients' limitations. The selection of the most appropriate measure must be made based on the psychometric properties [50]. In a review, González-Seguel et al. [51] identified sixty physical function measurement instruments under different domains for adult patients admitted to the ICU. Mobility was the most frequent domain to be measured and included 38 instruments. Some of the scoring systems for assessing the effectiveness of early mobilization on mobility in the ICU are mentioned in Table 4 [52-59].

3.4. Adverse Effects and Safety during Mobilization. Desaturation, heart rate elevation over $20 \%$, postural hypotension, unplanned extubation, tachypnea, agitation, discomfort, dislodgement of devices, and falls are some of the adverse events in different studies summarized in a systematic review [60].

There is a low frequency of adverse events associated with early mobilization of patients in the ICU $(\leq 4 \%)$ and most of them being nonthreatening. Even then, there are possibilities that mobilization may be withheld due to the concern of adverse effects [61]. Assessment before any mobilization session is necessary for the safety of the patient and for minimizing risk due to adverse events. Assessment of 
TABLE 3: Outcome measures for assessing the effectiveness of early mobilization in the intensive care unit.

\begin{tabular}{lc}
\hline Outcomes & Outcome measures \\
Muscle strength & $\begin{array}{c}\text { Hand-held dynamometer } \\
\text { Medical Research Council Score }\end{array}$ \\
\hline & Incidence of intensive care unit-acquired weakness (ICUAW) at hospital discharge \\
Physical function & ICU Mobility Scale \\
& Surgical intensive care unit optimal mobility score (SOMS) \\
& $\begin{array}{c}\text { Interval scores Physical Function ICU Test (PFIT-s) } \\
\text { Short Physical Performance Battery score (SPPB) } \\
\text { Functional Independence Measure (FIM) } \\
\text { Barthel Index }\end{array}$ \\
\hline Quality of life & Six-minute walk test \\
\hline
\end{tabular}

TABLE 4: Outcome measures for assessing the effectiveness of early mobilization on mobility in the intensive care unit.

\begin{tabular}{|c|c|c|c|c|}
\hline Author (year) & Outcome measure & $\begin{array}{l}\text { No. of } \\
\text { items }\end{array}$ & Total score & Psychometric properties \\
\hline Tipping et al. [53] (2016) & ICU Mobility Scale & 11 & $0-10$ & $\begin{array}{c}\text { Valid } \\
\text { Responsive } \\
\text { Acceptable floor and the } \\
\text { ceiling effect }\end{array}$ \\
\hline $\begin{array}{l}\text { Perme et al. [52] (2014) } \\
\text { Kawaguchi et al. [54] (2016) }\end{array}$ & Perme ICU Mobility Score & 15 & $\begin{array}{l}\qquad 0-32 \\
\text { Higher score-few potential mobility } \\
\text { restrictions and decreased assistance } \\
\text { Lower score-more potential } \\
\text { restrictions to mobility and more } \\
\text { assistance needed for mobility }\end{array}$ & $\begin{array}{c}\text { Valid } \\
\text { High reliability }(\alpha>0.90)\end{array}$ \\
\hline Corner et al. [55] (2014) & $\begin{array}{l}\text { Chelsea Critical Care } \\
\text { Physical Assessment tool }\end{array}$ & 10 & $0-50$ & $\begin{array}{l}\text { Valid } \\
\text { Limited floor and the } \\
\text { ceiling effect }\end{array}$ \\
\hline Denehy et al. [56] (2013) & $\begin{array}{c}\text { Interval scores } \\
\text { Physical Function ICU Test } \\
\text { (PFIT-s) }\end{array}$ & 4 & $0-12$ & $\begin{array}{c}\text { Valid } \\
\text { MCID }=1.5 \text { points }(\text { on } \\
\text { interval of } 10)\end{array}$ \\
\hline $\begin{array}{l}\text { Thrush et al. [57] (2012) } \\
\text { Huang et al. [58] (2016) }\end{array}$ & $\begin{array}{l}\text { Functional status score for } \\
\text { intensive care unit (FSS- } \\
\text { ICU) }\end{array}$ & 5 & $\begin{array}{l}\text { Higher the score, better the physical } \\
\text { functioning }\end{array}$ & $\begin{array}{c}\text { Valid } \\
\text { Responsive } \\
\text { Good internal } \\
\text { consistency } \\
\text { MCID }=2-5\end{array}$ \\
\hline Kasotakis et al. [59] (2012) & $\begin{array}{l}\text { Surgical intensive care unit } \\
\text { optimal mobility score } \\
\text { (SOMS) }\end{array}$ & 5 & $\begin{array}{l}\quad 0-4 \\
\text { Higher the score, better the mobility }\end{array}$ & $\begin{array}{l}\text { Valid } \\
\text { Reliable }\end{array}$ \\
\hline
\end{tabular}

TABLE 5: Safety measures for early mobilization in the intensive care unit.

\begin{tabular}{|c|c|c|c|}
\hline $\begin{array}{l}\text { Respiratory } \\
\text { considerations }\end{array}$ & Cardiovascular considerations & Neurological considerations & Others \\
\hline $\begin{array}{l}\text { (i) Peripheral oxygen } \\
\text { saturation }>88 \% \\
\text { (ii) Respiratory rate } \\
>5 \mathrm{bpm} \\
\text { (iii) }<40 \mathrm{bpm} \\
\text { (iv) } \mathrm{FiO}_{2}<0.6 \\
\text { (v) } \mathrm{PEEP}_{10}<\mathrm{cm} \mathrm{H}_{2} \mathrm{O} \\
\text { (vi) Airway protection }\end{array}$ & $\begin{array}{l}\text { (i) Heart rate }>40 \mathrm{bpm} \text { and }<130 \mathrm{bpm} \\
\text { (ii) Systolic blood pressure }<180 \mathrm{~mm} \\
\mathrm{Hg}>90 \mathrm{~mm} \mathrm{Hg} \\
\text { (iii) Mean arterial pressure }>60 \text { or }<110 \mathrm{~mm} \\
\mathrm{Hg} \\
\text { (iv) No vasoactive medications } \\
\text { (v) No increase in the dose of vasopressor in } \\
\text { the past two hours } \\
\text { (vi) No myocardial ischemia } \\
\text { (vii) No arrhythmia } \\
\text { (viii) No repetition of antiarrhythmic } \\
\text { medications }\end{array}$ & $\begin{array}{l}\text { (i) Level of consciousness, } \\
\text { no agitation } \\
\text { (ii) Not in coma } \\
\text { (iii) Following commands } \\
\text { (iv) Delirium } \\
\text { (v) Intracranial } \\
\text { pressure-not elevated }\end{array}$ & $\begin{array}{l}\text { (i) No unstable fracture or bony } \\
\text { instability } \\
\text { (ii) Not under continuous } \\
\text { hemodialysis } \\
\text { (iii) No deep vein thrombosis } \\
\text { (iv) Body temperature }<38.5^{\circ} \\
\text { (v) No active bleeding }\end{array}$ \\
\hline
\end{tabular}

$\mathrm{FiO}_{2}$, fraction of inspired oxygen; PEEP, positive end-expiratory pressure. 
TABLE 6: Red signals for active mobilization of mechanically ventilated patients.

= red signal Exercise in bed Exercise outside bed

Percutaneous oxygen saturation $<90 \%$

High frequency oscillatory mode of ventilation

Prone positioning

Intravenous hypertensive therapy for emergency hypertension

Bradycardia requiring pharmacological intervention or awaiting pacemaker insertion

Mean arterial pressure below the target range

Dependent rhythm on a transvenous or epicardial pacemaker

Stable tachycardia with a ventricular rate $>150 \mathrm{bpm}$

Intraaortic balloon pump

Extracorporeal membrane oxygen

Cardiac ischemia (ongoing chest pain)

Unarousable or deeply sedated patient: RASS $<-2$

Very agitated or combative patient: RASS $>+2$

Active management of intracranial hypertension and raised intracranial pressure

Open lumbar drain (unclamped)

Uncontrolled seizures

Unstable/unstabilized major fractures

Large exposed surgical wound

Known uncontrolled active hemorrhage

Femoral sheath

RASS, Richmond Agitation-Sedation Scale.

TABLE 7: Green signals for active mobilization of mechanically ventilated patients.

\begin{tabular}{|c|c|c|}
\hline$\checkmark=$ green signal & $\begin{array}{l}\text { Exercise in } \\
\text { bed }\end{array}$ & $\begin{array}{l}\text { Exercise outside } \\
\text { bed }\end{array}$ \\
\hline Endotracheal tube & $\checkmark$ & $\checkmark$ \\
\hline Tracheostomy tube & $\checkmark$ & $\checkmark$ \\
\hline Fraction of inspired oxygen $\leq 0.6$ & $\checkmark$ & $\checkmark$ \\
\hline Percutaneous oxygen saturation $\geq 90 \%$ & $\checkmark$ & $\checkmark$ \\
\hline Respiratory rate $\leq 30 \mathrm{bpm}$ & $\checkmark$ & $\checkmark$ \\
\hline PEEP $\leq 10 \mathrm{~cm} \mathrm{H}_{2} \mathrm{O}$ & $\checkmark$ & $\checkmark$ \\
\hline $\begin{array}{l}\text { Mean arterial pressure more than the lower limit of target range while receiving no support or low } \\
\text { level of support }\end{array}$ & $\checkmark$ & $\checkmark$ \\
\hline Stable underlying rhythm with a transvenous or epicardial pacemaker & $\checkmark$ & $\checkmark$ \\
\hline Femoral intraaortic balloon pump & $\checkmark$ & \\
\hline Ventricular assist device & $\checkmark$ & $\checkmark$ \\
\hline Extracorporeal membrane oxygenation: femoral or subclavian & $\checkmark$ & \\
\hline Pulmonary artery catheterization or other continuous cardiac monitors & $\checkmark$ & \\
\hline Known or suspected severe aortic stenosis & $\checkmark$ & \\
\hline Drowsy, calm, or restless patient: RASS -1 to +1 & $\checkmark$ & $\checkmark$ \\
\hline Delirium tool negative & $\checkmark$ & $\checkmark$ \\
\hline Delirium tool positive and able to obey simple instructions & $\checkmark$ & \\
\hline Craniectomy & $\checkmark$ & \\
\hline Lumbar drain (unclamped) & $\checkmark$ & \\
\hline Acute spinal cord injury & $\checkmark$ & \\
\hline Subarachnoid bleed with unclipped aneurysm & $\checkmark$ & \\
\hline Large open surgical wound & $\checkmark$ & \\
\hline Suspicion or increased risk of active hemorrhage & $\checkmark$ & \\
\hline Intensive care unit-acquired weakness & $\checkmark$ & $\checkmark$ \\
\hline Continuous renal replacement therapies & $\checkmark$ & $\checkmark$ \\
\hline Arterial and venous femoral catheters & $\checkmark$ & $\checkmark$ \\
\hline Other attachment and drains & $\checkmark$ & $\checkmark$ \\
\hline
\end{tabular}

PEEP, positive end-expiratory pressure; RASS, Richmond Agitation-Sedation Scale.

whether a patient should be mobilized or not can be aided by objective criteria that ensures safety $[60,61]$.

A review on safety criteria for starting early mobilization was conducted by Albanaz da Conceição et al. [60]. The safety criteria included parameters that were categorized into groups, which included cardiovascular, respiratory, neurological, and others. It is described in Table 5.

Hodgson et al. [61] developed safety measures for the active mobilization of patients with critical illness who are mechanically ventilated. They used a color-coded system for 
TABLE 8: Criteria for termination of mobilization.

(i) Tachycardia ( $>140$ beats/min)

(ii) Bradycardia ( $<50$ beats/min)

(iii) Arrhythmias

(iv) Hypertension - systolic blood pressure $>180 \mathrm{~mm} \mathrm{Hg}$

(v) Hypotension-systolic blood pressure $<80 \mathrm{~mm} \mathrm{Hg}$

(vi) Symptomatic orthostatic hypotension

(vii) Mean arterial pressure $<60$ or $>110 \mathrm{~mm} \mathrm{Hg}$

(viii) Oxygen saturation $<88 \%$

(ix) Asynchrony with mechanical ventilation

(x) Abnormality in respiratory rate $->40$ breaths $/ \mathrm{min}$ or $<5$ breaths/min

(xi) Significant use of accessory muscles

(xii) Significant chest pain

(xiii) Excessive pallor or flushing of the skin

(xiv) Extreme fatigue

(xv) Patient's intolerance or request to stop

(xvi) Hemorrhage and unexpected removal of medical devices such as the chest tube, endotracheal tube, feeding tube, abdominal drain, urinary catheter, arterial catheter, hemodialysis catheter, or venous catheter

TABle 9: Practice of early mobilization.

\begin{tabular}{|c|c|c|c|}
\hline Study (year) & Study design & Place & Population \\
\hline $\begin{array}{l}\text { Timenetsky et al. [69] } \\
(2020)\end{array}$ & $\begin{array}{c}\text { 1-day point prevalence } \\
\text { study }\end{array}$ & Brazil & $\begin{array}{l}348 \text { adult patients with more than } \\
24 \text { h of ICU stay ( } 24 \text { mixed ICU, } 1 \\
\text { surgical ICU, and } 1 \text { medical ICU) }\end{array}$ \\
\hline
\end{tabular}

Sibilla et al. [70] (2017) Point prevalence study Switzerland 161 mechanically ventilated patients from 35 ICUs

High prevalence of mobilization activities in critically ill patients Not much active mobilization in mechanically ventilated patients Only $33 \%$ of the mechanically ventilated patients actively mobilized

\begin{tabular}{lccr}
\hline Nydahl et al. [71] (2014) & $\begin{array}{c}\text { 1-day point prevalence } \\
\text { study }\end{array}$ & Germany & Mechanically ventilated patients \\
\hline Berney et al. [72] (2013) & $\begin{array}{c}\text { One-day point } \\
\text { prevalence study }\end{array}$ & $\begin{array}{c}\text { Australia and } \\
\text { New Zealand }\end{array}$ & $\begin{array}{c}514 \text { patients admitted to the } \\
\text { intensive care unit from 38 ICUs }\end{array}$ \\
\hline $\begin{array}{l}\text { TEAM study } \\
\text { investigators [65] (2015) }\end{array}$ & Cohort study & $\begin{array}{c}\text { Australia and } \\
\text { New Zealand }\end{array}$ & $\begin{array}{r}192 \text { mechanically ventilated ICU } \\
\text { patients from 12 ICUs }\end{array}$ \\
\hline
\end{tabular}

Three quarters of the patients not mobilized out of bed

Low patient mobilization on that day

$84 \%$ of the physiotherapy sessions did not include early mobilization Mobilizing patient three times and above per shift was reported by $75 \%$ of nurses. $47.7 \%$ reported that they only performed passive

Cross-sectional survey

Leong et al. [73] (2017) on early mobilization of mechanically ventilated patients

Malaysia

186 nurses working in adult critical care units of University Malaya Medical Centre (UMMC), a 1200-bed referral centre range of motion to mechanically ventilated patients. $29.5 \%$ reported that they only provide active ROM for their patient. $72 \%$ nurses reported that they had not gone through patient mobilization training

97.6\% participants reported that patients received mobilization in some form. Mobilization in neurological intensive care units of various forms practiced in the neurological ICUs of India. Less availability of physiotherapists on weekends and night hours.

659 physicians of the Indian Society of Critical Care Medicine and the Indian Society of Anesthesiologists who worked full time or part time in intensive care
High awareness of benefits of early mobilization and low implementation 
TABLE 10: Barriers to early mobilization.

\begin{tabular}{|c|c|}
\hline Author (year) & Reported barriers \\
\hline $\begin{array}{l}\text { Anekwe et al. [81] } \\
\text { (2017) }\end{array}$ & $\begin{array}{l}\text { Perceived patient level barriers } \\
\text { (i) Medical instability } \\
\text { (ii) Risk of dislodgement } \\
\text { (iii) Excessive sedation } \\
\text { (iv) Endotracheal intubation } \\
\text { (v) Cognitive impairment } \\
\text { (vi) Inadequate analgesia } \\
\text { Perceived institutional level barriers } \\
\text { (i) Orders required } \\
\text { (ii) Lack of equipment } \\
\text { Perceived provider level barriers } \\
\text { (i) Limited staff } \\
\text { (ii) Communication among providers } \\
\text { (iii) Inadequate training } \\
\text { (iv) Not a priority } \\
\text { (v) Safety concerns }\end{array}$ \\
\hline Costa et al. [82] (2017) & $\begin{array}{l}\text { Patient related } \\
\text { (i) Lack of patient's cooperation } \\
\text { (ii) Patient's instability and safety concerns } \\
\text { (iii) Patient status issues (fatigue, diarrhea, leaking wound, weight size, confusion, agitation, and death) } \\
\text { Clinician related } \\
\text { (i) Lack of awareness and knowledge about the protocol } \\
\text { (ii) Lack of conceptual agreement with guidelines } \\
\text { (iii) Lack of self-efficacy and confidence in protocol implementation } \\
\text { (iv) Staff and patient safety concerns } \\
\text { (v) The perception that rest equals healing } \\
\text { (vi) Reluctance to follow protocol (due to previous adverse outcomes) } \\
\text { (vii) Lack of confidence } \\
\text { (viii) Perceived workload } \\
\text { (ix) Safety of tubes, wires, and catheters } \\
\text { Protocol related } \\
\text { (i) Unavailability of protocol } \\
\text { (ii) Unclear protocol criteria } \\
\text { (iii) Protocol development cost (money and time) } \\
\text { (iv) Learning curve (possibility for the clinician to test guideline and observe other clinicians using the guideline } \\
\text { easily) } \\
\text { (v) Lack of clarity as to who is responsible, steps needed to take, and expected standards for protocol } \\
\text { implementation } \\
\text { (vi) Lack of confidence in evidence supporting protocol and guideline developer } \\
\text { (vii) Lack of confidence in the reliability of screening tools } \\
\text { ICU contextual barriers culture } \\
\text { (i) Interprofessional team care coordination, communication, and collaboration barriers } \\
\text { (ii) Lack of leadership/management } \\
\text { (iii) Interprofessional clinician staffing, workload, and time } \\
\text { (iv) Physical environment, equipment, and resources } \\
\text { (v) Staff turnover } \\
\text { (vi) Low prioritization and perceived importance } \\
\text { (vii) Scheduling conflicts (i- +-e, patient off, at dialysis, and procedure) }\end{array}$ \\
\hline
\end{tabular}

the criteria. The red signal indicated a significant risk of adverse events. The yellow signal indicated that mobilization was possible but only after measuring risk versus benefit and there could be a potential risk of adverse events. The green signal indicated that active mobilization could be performed with a low risk of adverse events. The red and green signals are mentioned in Tables 6 and 7.

3.5. Criteria for Termination of Early Mobilization. Safety criteria also include termination criteria that indicate discontinuation of the early mobilization session and allow the patients to rest [61]. Studies performed by Liu et al. [62] and Perme and Chandrashekar [63] have suggested criteria for termination of mobilization, which are stated in Table 8.

3.6. Practice of Early Mobilization Globally. Early mobilization is an intervention that reports positive outcomes, is considered safe to be practiced, and has safety and termination criteria [64-66]. Various studies have developed protocols for early mobilization in different ICUs which have been found to be effective, safe, and practicable. These 
TABLE 11: Strategies for overcoming barriers.

Barriers Strategies

Patient-related barriers

(i) Hemodynamic instability

(ii) Pain

(iii) Deep sedation

(iv) Agitation and delirium

(v) Patient denial, lacking motivation

(vi) ICU equipment and devices

Structural barriers

(i) Limited staff

(ii) Lack of protocols and limited guidelines

(iii) Limited equipment
(i) Stepwise approach
(ii) Pain management before mobilization
(iii) Regular assessment, lighter sedation
(iv) Assessment, antipsychotic medications
(v) Patient education and encouragement
(vi) Portable devices, secure lines, drains, and interdisciplinary teamwork

(i) Additional staff, independent mobility team

(ii) Develop protocols, safety criteria

(iii) Training for appropriate use of equipment, financial, and the cost analysis model of economic benefit

(i) Promotion of mobility programs

(ii) Interprofessional education

(i) Lack of mobilization culture

(ii) Early mobilization, not a priority

Process-related barriers

(i) A dearth of coordination and planning

(ii) Risks for mobility providers (i) Regular screening of patients, interprofessional coordination, and planning

(ii) Training, appropriate equipment, and mobility team protocols include different levels of mobilization consisting of activities such as passive or active range of motion exercises, positioning, sitting upright on the bed, sitting on edge of bed, standing, sitting on chair, and walking and are applicable to patients with or without mechanical ventilation $[29,36,67,68]$. Even then, early mobilization is not practiced commonly. Studies on the practice of early mobilization in different countries are mentioned in Table 9 [65, 69-75].

3.7. Barriers to Early Mobilization. The practice of early mobilization is still not common in the clinical setting due to different perceived barriers [76, 77]. Some of these barriers include hemodynamic instability, presence of vascular attachments, altered sleep patterns, safety of the patients, lack of communication and teamwork between various professionals, lack of professionals, inadequate time, delirium, extreme sedation, risk of musculoskeletal injury, and extreme stress at work $[13,78,79]$.

Leditschke et al. [80] reported modifiable and nonmodifiable barriers to early mobilization. Modifiable barriers comprised vascular access catheters in a femoral position, sedation management, timing of procedures, agitation, and low Glasgow Coma Score. Nonmodifiable factors comprised hemodynamic instability, respiratory instability, neurologic instability (difficulty in controlling intracranial hypertension), and medical orders. According to Sibilla et al. [70], less-perceived barriers were encountered during passive mobilization when compared to patients who were mobilized actively. Various barriers to early mobilization are mentioned in Table 10 [81, 82].

3.8. Strategies to Overcome Barriers. Having an understanding of barriers for early mobilization and developing strategies to overcome those assist professionals in practicing early mobilization as part of daily clinical practice. A study by Dubb et al. [83] merged the available data on early mobilization barriers and strategies to overcome them. A few of them are stated in Table 11.

\section{Conclusion}

Early mobilization is found to have positive effects such as decreasing muscle atrophy, mechanical ventilation duration, length of hospital stay, and increasing functional capacity but do not have an impact on long-term outcomes. Early mobilization-in different intensive care units, namely, surgical, cardiac, and neurological ICU-has been studied and found to be effective. As suggested by most of the systematic reviews, further good quality studies need to be conducted. Also, mobilization protocols need to be compared to find the most effective protocol. Newer techniques such as electrical muscle stimulation, cycling, and hydrotherapy are safe and found to have some positive outcomes. Sara Combilizer can be used safely to facilitate early mobilization. More randomized controlled trials need to be performed to confirm the findings of the existing studies. Outcome measures, specific to the ICU, are available and should be used to quantify patients' status at different intervals of time and to identify achievements due to early mobilization.

Safety criteria, with various physiological considerations, for in-bed and outside bed mobilization along with criteria for termination have been mentioned by various authors and can be used for safe practice. Although early mobilization has benefits, it is not commonly practiced in many countries. Various barriers to early mobilization, which include patient-related, institution-related, and clinician-related barriers have been identified, and different strategies have been used to overcome them to allow the smooth practice of early mobilization. 


\section{Conflicts of Interest}

The authors declare there are no conflicts of interest.

\section{Acknowledgments}

The authors would like to thank Dr. Madhusudan Upadya, Professor, Department of Anaesthesia, KMC Mangalore, Manipal Academy of Higher Education, Dr. Alfred Augustine, Professor, Department of Surgery, KMC Mangalore, Manipal Academy of Higher Education, Ms. Manjiri Kulkarni, Ms. Tiala Jane Lewis, and Mrs. Kripa Gatty for their timely help and valuable suggestions.

\section{References}

[1] F. E. Udwadia, "Overview," in Principles of Critical Care, F. E. Udwadia, Ed., p. 3, 3rd edition, Jaypee Brothers Medical Publishers (P) Ltd., New Delhi, India, 2014.

[2] R. G. Brower, "Consequences of bed rest," Critical Care Medicine, vol. 37, no. 10, pp. 422-428, 2009.

[3] C. L. Rochester, "Rehabilitation in the intensive care unit," Seminars in Respiratory and Critical Care Medicine, vol. 30, no. 6, pp. 656-669, 200.

[4] D. M. Needham, "Mobilizing patients in the intensive care unit," JAMA, vol. 300, no. 14, pp. 1685-1690, 2008.

[5] S. V. Desai, T. J. Law, and D. M. Needham, "Long-term complications of critical care," Critical Care Medicine, vol. 39, no. 2, pp. 371-379, 2011.

[6] R. Topp, M. Ditmyer, K. King, K. Doherty, and J. Hornyak III, "The effect of bed rest and potential of prehabilitation on patients in the intensive care unit," AACN Clinical Issues: Advanced Practice in Acute and Critical Care, vol. 13, no. 2, pp. 263-276, 2002.

[7] M. A. Kumar, F. G. Romero, and K. Dharaneeswaran, "Early mobilization in neurocritical care patients," Current Opinion in Critical Care, vol. 26, no. 2, pp. 147-154, 2020.

[8] E. Ries, "Some radical changes in the after-treatment of celiotomy cases," JAMA: The Journal of the American Medical Association, vol. 33, no. 8, pp. 454-456, 1899.

[9] H. J. Epstein and A. J. Fleischer, "The disadvantages of the prolonged period of postpartum rest in bed," American Journal of Obstetrics and Gynecology, vol. 14, no. 3, pp. 360363, 1927.

[10] M. D. Hashem, A. Nelliot, and D. M. Needham, "Early mobilization and rehabilitation in the ICU: moving back to the future," Respiratory Care, vol. 61, no. 7, pp. 971-979, 2016.

[11] J. R. Burns and F. L. Jones, "Early ambulation of patients requiring ventilatory assistance," Chest, vol. 68, no. 4, p. 608, 1975.

[12] G. Foss, "A method for augmenting ventilation during ambulation," Physical Therapy, vol. 52, no. 5, pp. 519-520, 1972.

[13] C. L. Hodgson, S. Berney, M. Harrold, M. Saxena, and R. Bellomo, "Clinical review: early patient mobilization in the ICU," Critical Care (London, England), vol. 17, no. 1, p. 207, 2013.

[14] C. Clarissa, L. Salisbury, S. Rodgers, and S. Kean, "Early mobilisation in mechanically ventilated patients: a systematic integrative review of definitions and activities," Journal of Intensive Care, vol. 7, no. 1, 2019.

[15] B. F. Olkowski, M. A. Devine, L. E. Slotnick et al., "Safety and feasibility of an early mobilization program for patients with aneurysmal subarachnoid hemorrhage," Physical Therapy, vol. 93, no. 2, pp. 208-215, 2013.

[16] E. Dean and S. Butcher, "Mobilization and exercise: physiological basis for assessment, evaluation, and training," in Cardiovascular and Pulmonary Physical Therapy Evidence to Practice, D. Frownfelter and E. Dean, Eds., pp. 244-272, Elsevier, Alpharetta, GA, USA, 5th edition, 2012.

[17] G. Zhang, K. Zhang, W. Cui, Y. Hong, and Z. Zhang, "The effect of early mobilization for critical ill patients requiring mechanical ventilation: a systematic review and meta-analysis," Journal of Emergency and Critical Care Medicine, vol. 2, p. 9, 2018.

[18] L. Zhang, W. Hu, Z. Cai et al., "Early mobilization of critically ill patients in the intensive care unit: a systematic review and meta-analysis," PLoS One, vol. 14, no. 10, Article ID e0223185, 2019.

[19] K. A. Doiron, T. C. Hoffmann, and E. M. Beller, "Early intervention (mobilization or active exercise) for critically ill adults in the intensive care unit," The Cochrane Database of Systematic Reviews, vol. 3, Article ID CD010754, 2018.

[20] K. Zang, B. Chen, M. Wang et al., "The effect of early mobilization in critically ill patients: a meta-analysis," Nursing in Critical Care, pp. 1-8, 2019.

[21] C. J. Tipping, M. Harrold, A. Holland, L. Romero, T. Nisbet, and C. L. Hodgson, "The effects of active mobilisation and rehabilitation in ICU on mortality and function: a systematic review," Intensive Care Medicine, vol. 43, no. 2, pp. 171-183, 2016.

[22] A. C. Castro-Avila, P. Serón, E. Fan, M. Gaete, and S. Mickan, "Effect of early rehabilitation during intensive care unit stay on functional status: systematic review and meta-analysis," PLoS One, vol. 10, no. 7, Article ID e0130722, 2015.

[23] Y. Okada, T. Unoki, Y. Matsuishi, Y. Egawa, K. Hayashida, and S. Inoue, "Early versus delayed mobilization for inhospital mortality and health-related quality of life among critically ill patients: a systematic review and meta-analysis," Journal of Intensive Care, vol. 7, no. 1, pp. 1-9, 2019.

[24] O. R. Amundadottir, R. J. Jónasdóttir, K. Sigvaldason et al., "Effects of intensive upright mobilisation on outcomes of mechanically ventilated patients in the intensive care unit: a randomised controlled trial with 12-months follow-up," European Journal of Physiotherapy, pp. 1-11, 2019.

[25] L. Denehy, E. H. Skinner, L. Edbrooke et al., "Exercise rehabilitation for patients with critical illness: a randomized controlled trial with 12 months of follow-up," Critical Care, vol. 17, no. 4, p. R156, 2013.

[26] S. E. Wright, K. Thomas, G. Watson et al., "Intensive versus standard physical rehabilitation therapy in the critically ill (EPICC): a multicentre, parallel-group, randomised controlled trial," Thorax, vol. 73, no. 3, pp. 213-221, 2017.

[27] T. Castelino, J. F. Fiore, P. Niculiseanu, T. Landry, B. Augustin, and L. S. Feldman, "The effect of early mobilization protocols on postoperative outcomes following abdominal and thoracic surgery: a systematic review," Surgery, vol. 159, no. 4, pp. 991-1003, 2016.

[28] S. J. Schaller, M. Anstey, M. Blobner et al., "Early, goal-directed mobilisation in the surgical intensive care unit: a randomised controlled trial," The Lancet, vol. 388, no. 10052, pp. 1377-1388, 2016.

[29] M. Zomorodi, D. Topley, and M. McAnaw, "Developing a mobility protocol for early mobilization of patients in a surgical/trauma ICU," Critical Care Research and Practice, vol. 2012, Article ID 964547, 10 pages, 2012. 
[30] G. Soares, D. Ferreira, M. Gonçalves et al., "Prevalence of major postoperative complications in cardiac surgery," International Journal of Cardiovascular Sciences, vol. 24, no. 3, pp. 139-146, 2011.

[31] S. T. Moradian, M. Najafloo, H. Mahmoudi, and M. S. Ghiasi, "Early mobilization reduces the atelectasis and pleural effusion in patients undergoing coronary artery bypass graft surgery: a randomized clinical trial," Journal of Vascular Nursing, vol. 35, no. 3, pp. 141-145, 2017.

[32] P. M. R. Santos, N. A. Ricci, É. A. B. Suster, D. M. Paisani, and L. D. Chiavegato, "Effects of early mobilisation in patients after cardiac surgery: a systematic review," Physiotherapy, vol. 103, no. 1, pp. 1-12, 2017.

[33] K. Klein, M. Mulkey, J. F. Bena, and N. M. Albert, "Clinical and psychological effects of early mobilization in patients treated in a neurologic ICU," Critical Care Medicine, vol. 43, no. 4, pp. 865-873, 2015.

[34] A. Rocca, J. Pignat, L. Berney et al., "Sympathetic activity and early mobilization in patients in intensive and intermediate care with severe brain injuries: a preliminary prospective randomized study," BMC Neurology, vol. 16, no. 1, p. 169, 2016.

[35] A. Sundseth, B. Thommessen, and O. M. Rønning, "Early mobilization after acute stroke," Journal of Stroke and Cerebrovascular Diseases, vol. 23, no. 3, pp. 496-499, 2014.

[36] M. Alamri, I. Waked, F. Amin, K. Al-quliti, and M. Manzar, "Effectiveness of an early mobility protocol for stroke patients in intensive care unit," Neurosciences, vol. 24, no. 2, pp. 81-88, 2019.

[37] K. Diserens, T. Moreira, L. Hirt et al., "Early mobilization out of bed after ischaemic stroke reduces severe complications but not cerebral blood flow: a randomized controlled pilot trial," Clinical Rehabilitation, vol. 26, no. 5, pp. 451-459, 2011.

[38] M. Bartolo, S. Bargellesi, C. Castioni et al., "Mobilization in early rehabilitation in intensive care unit patients with severe acquired brain injury: an observational study," Journal of Rehabilitation Medicine, vol. 49, no. 9, pp. 715-722, 2017.

[39] L. F. Falavigna, M. G. Silva, A. L. de Almeida Freitas et al., "Effects of electrical muscle stimulation early in the quadriceps and tibialis anterior muscle of critically ill patients," Physiotherapy Theory and Practice, vol. 30, no. 4, pp. 223-228, 2013.

[40] M. V. Baron, M. V. d. M. Pinto, J. Koepp et al., "Neuromuscular electrical stimulation in intensive care unit patients: integrative review," Modern Research in Inflammation, vol. 8, no. 2, pp. 11-27, 2019.

[41] A. Acqua, A. Sachetti, L. Santos et al., "Use of neuromuscular electrical stimulation to preserve the thickness of abdominal and chest muscles of critically ill patients: a randomized clinical trial," Journal of Rehabilitation Medicine, vol. 49, no. 1 , pp. 40-48, 2017.

[42] A. Fischer, M. Spiegl, K. Altmann et al., "Muscle mass, strength and functional outcomes in critically ill patients after cardiothoracic surgery: does neuromuscular electrical stimulation help? The catastim 2 randomized controlled trial," Critical Care, vol. 20, no. 1, p. 30, 2016.

[43] G. G. Gama Lordello, G. G. Gonçalves Gama, G. Lago Rosier, P. A. D. d. C. Viana, L. C. Correia, and L. E. Fonteles Ritt, "Effects of cycle ergometer use in early mobilization following cardiac surgery: a randomized controlled trial," Clinical Rehabilitation, vol. 34, no. 4, pp. 450-459, 2020.

[44] A. d. S. Machado, R. C. Pires-Neto, M. T. X. Carvalho, J. C. Soares, D. M. Cardoso, and I. M. d. Albuquerque, "Effects that passive cycling exercise have on muscle strength, duration of mechanical ventilation, and length of hospital stay in critically ill patients: a randomized clinical trial," Jornal Brasileiro de Pneumologia, vol. 43, no. 2, pp. 134-139, 2017.

[45] L. Santos, A. Dall' Acqua, A. Sachetti et al., "Use of electrical neuromuscular stimulation to preserve the morphology of abdominal and chest muscles of critical patients: randomized clinical trial," Intensive Care Medicine Experimental, vol. 3, no. 1, p. A552, 2015.

[46] G. Fossat, F. Baudin, L. Courtes et al., "Effect of in-bed leg cycling and electrical stimulation of the quadriceps on global muscle strength in critically ill adults," JAMA, vol. 320, no. 4, pp. 368-378, 2018.

[47] D. McWilliams, G. Atkins, J. Hodson, and C. Snelson, "The sara combilizer as an early mobilisation aid for critically ill patients: a prospective before and after study," Australian Critical Care, vol. 30, no. 4, pp. 189-195, 2017.

[48] K. M. Felten-Barentsz, A. J. C. Haans, A. S. Slutsky, L. M. A. Heunks, and J. G. van der Hoeven, "Feasibility and safety of hydrotherapy in critically ill ventilated patients," American Journal of Respiratory and Critical Care Medicine, vol. 191, no. 4, pp. 476-477, 2015.

[49] E. Finch, D. Brooks, P. Stratford, and N. Mayo, Physical Rehabilitation Outcome Measures, Canadian Physiotherapy Association, Ottawa, Canada, 2nd edition, 2002.

[50] S. Parry, L. Denehy, L. Beach, S. Berney, H. Williamson, and C. Granger, "Functional outcomes in ICU-what should we be using? - an observational study," Critical Care, vol. 19, no. 1, p. 127, 2015.

[51] F. González-Seguel, E. J. Corner, and C. Merino-Osorio, "International classification of functioning, disability, and health domains of 60 physical functioning measurement instruments used during the adult intensive care unit stay: a scoping review," Physical Therapy, vol. 99, no. 5, pp. 627-640, 2019.

[52] C. Perme, R. K. Nawa, C. Winkelman, and F. Masud, "A tool to assess mobility status in critically ill patients: the perme intensive care unit mobility score," Methodist DeBakey Cardiovascular Journal, vol. 10, no. 1, pp. 41-49, 2014.

[53] C. J. Tipping, M. J. Bailey, R. Bellomo et al., "The ICU mobility scale has construct and predictive validity and is responsive. A multicenter observational study," Annals of the American Thoracic Society, vol. 13, no. 6, pp. 887-893, 2016.

[54] Y. M. F. Kawaguchi, R. Nawa, R. K. Nawa, T. B. Figueiredo, L. Martins, and R. C. Pires-Neto, "Perme intensive care unit mobility score and ICU mobility scale: translation into Portuguese and cross-cultural adaptation for use in Brazil," Jornal Brasileiro de Pneumologia, vol. 42, no. 6, pp. 429-434, 2016.

[55] E. J. Corner, N. Soni, J. M. Handy, and S. J. Brett, "Construct validity of the Chelsea critical care physical assessment tool: an observational study of recovery from critical illness," Critical Care, vol. 18, no. 2, p. R55, 2014.

[56] L. Denehy, N. A. de Morton, E. H. Skinner et al., "A physical function test for use in the intensive care unit: validity, responsiveness, and predictive utility of the physical function ICU test (scored)," Physical Therapy, vol. 93, no. 12, pp. 1636-1645, 2013.

[57] A. Thrush, M. Rozek, and J. L. Dekerlegand, "The clinical utility of the functional status score for the intensive care unit (FSS-ICU) at a long-term acute care hospital: a prospective cohort study," Physical Therapy, vol. 92, no. 12, pp. 1536-1545, 2012. 
[58] M. Huang, K. Chan, J. Zanni et al., "Functional status score for the ICU," Critical Care Medicine, vol. 44, no. 12, pp. 1155-1164, 2016.

[59] G. Kasotakis, U. Schmidt, D. Perry et al., "The surgical intensive care unit optimal mobility score predicts mortality and length of stay," Critical Care Medicine, vol. 40, no. 4, pp. 1122-1128, 2012.

[60] T. Conceição, A. Gonzáles, F. Figueiredo, D. Vieira, and D. Bündchen, "Safety criteria to start early mobilization in intensive care units. Systematic review," Revista Brasileira de Terapia Intensiva, vol. 29, no. 4, pp. 509-519, 2017.

[61] C. Hodgson, K. Stiller, D. Needham et al., "Expert consensus and recommendations on safety criteria for active mobilization of mechanically ventilated critically ill adults," Critical Care, vol. 18, no. 6, p. 658, 2014.

[62] K. Liu, T. Ogura, K. Takahashi et al., "The safety of a novel early mobilization protocol conducted by ICU physicians: a prospective observational study," Journal of Intensive Care, vol. 6, no. 1, p. 10, 2018.

[63] C. Perme and R. Chandrashekar, "Early mobility and walking program for patients in intensive care units: creating a standard of care," American Journal of Critical Care, vol. 18, no. 3, pp. 212-221, 2009.

[64] J. Adler and D. Malone, "Early mobilization in the intensive care unit: a systematic review," Cardiopulmonary Physical Therapy Journal, vol. 23, no. 1, pp. 5-13, 2012.

[65] TEAM Study Investigators, "Early mobilization and recovery in mechanically ventilated patients in the ICU: a bi-national, multi-center, prospective cohort study," Critical Care, vol. 19, no. 1 , p. $81,2015$.

[66] Z. Li, X. Peng, B. Zhu, Y. Zhang, and X. Xi, "Active mobilization for mechanically ventilated patients: a systematic review," Archives of Physical Medicine and Rehabilitation, vol. 94, no. 3, pp. 551-561, 2013.

[67] P. E. Morris, A. Goad, C. Thompson et al., "Early intensive care unit mobility therapy in the treatment of acute respiratory failure," Critical Care Medicine, vol. 36, no. 8, pp. 2238-2243, 2008.

[68] M. Sigler, K. Nugent, R. Alalawi et al., "Making of a successful early mobilization program for a medical intensive care unit," Southern Medical Journal, vol. 109, no. 6, pp. 342-345, 2016.

[69] K. T. Timenetsky, A. S. Neto, M. S. Assunção et al., "Mobilization practices in the ICU: a nationwide 1-day pointprevalence study in Brazil," PLoS One, vol. 15, no. 4, Article ID e0230971, 2020.

[70] A. Sibilla, P. Nydahl, N. Greco et al., "Mobilization of mechanically ventilated patients in Switzerland," Journal of Intensive Care Medicine, vol. 35, no. 1, pp. 55-62, 2020.

[71] P. Nydahl, A. P. Ruhl, G. Bartoszek et al., "Early mobilization of mechanically ventilated patients," Critical Care Medicine, vol. 42, no. 5, pp. 1178-1186, 2014.

[72] S. C. Berney, M. Harrold, S. A. Webb et al., "Intensive care unit mobility practices in Australia and New Zealand: a point prevalence study," Critical Care and Resuscitation: Journal of the Australasian Academy of Critical Care Medicine, vol. 15, no. 4, pp. 260-265, 2013.

[73] Y. L. Leong, A. R. Rasnah, and M. C. Chong, "Patient early mobilization: Malaysia's study of nursing practices," Journal of Intensive and Critical Care, vol. 3, no. 3, p. 29, 2017.

[74] A. Bhat, K. Chakravarthy, and B. K. Rao, "Mobilization of patients in neurological intensive care units of India: a survey," Indian Journal of Critical Care Medicine, vol. 20, no. 6, pp. 337-341, 2016.
[75] R. Chawla, S. N. Myatra, N. Ramakrishnan, S. Todi, S. Kansal, and S. K. Dash, "Current practices of mobilization, analgesia, relaxants and sedation in Indian ICUs: a survey conducted by the Indian society of critical care medicine," Indian Journal of Critical Care Medicine: Peer-Reviewed, Official Publication of Indian Society of Critical Care Medicine, vol. 18, no. 9, pp. 575-584, 2014.

[76] M. D. Hashem, A. M. Parker, and D. M. Needham, "Early mobilization and rehabilitation of patients who are critically ill," Chest, vol. 150, no. 3, pp. 722-731, 2016.

[77] S. Jolley, J. Regan-Baggs, R. Dickson, and C. Hough, "Medical intensive care unit clinician attitudes and perceived barriers towards early mobilization of critically ill patients: a crosssectional survey study," BMC Anesthesiology, vol. 14, no. 1, p. 84, 2014.

[78] P. Fontela, L. Forgiarini Júnior, and G. Friedman, "Clinical attitudes and perceived barriers to early mobilization of critically ill patients in adult intensive care units," Revista Brasileira de Terapia Intensiva, vol. 30, no. 2, pp. 187-194, 2018.

[79] C. L. Harris and S. Shahid, "Physical therapy-driven quality improvement to promote early mobility in the intensive care unit," Baylor University Medical Center Proceedings, vol. 27, no. 3, pp. 203-207, 2014.

[80] A. I. Leditschke, M. Green, J. Irvine, B. Bissett, and I. A. Mitchell, "What are the barriers to mobilizing intensive care patients?" Cardiopulmonary Physical Therapy Journal, vol. 23, no. 1, pp. 26-29, 2012.

[81] D. E. Anekwe, K. K.-Y. Koo, M. de Marchie, P. Goldberg, D. Jayaraman, and J. Spahija, "Interprofessional survey of perceived barriers and facilitators to early mobilization of critically ill patients in montreal, Canada," Journal of Intensive Care Medicine, vol. 34, no. 3, pp. 218-226, 2017.

[82] D. K. Costa, M. R. White, E. Ginier et al., "Identifying barriers to delivering the awakening and breathing coordination, delirium, and early exercise/mobility bundle to minimize adverse outcomes for mechanically ventilated patients," Chest, vol. 152, no. 2, pp. 304-311, 2017.

[83] R. Dubb, P. Nydahl, C. Hermes et al., "Barriers and strategies for early mobilization of patients in intensive care units," Annals of the American Thoracic Society, vol. 13, no. 5, pp. 724-730, 2016. 